\title{
ON IDEALS OF RINGS OF FRACTIONS AND RINGS OF POLYNOMIALS
}

\author{
Yuan Ting NaI AND Dongsheng ZhaO
}

\begin{abstract}
We investigate the links between the lattice $\operatorname{Idl}(R)$ of ideals of a commutative ring $R$ and the lattices $\operatorname{Idl}\left(R^{\prime}\right)$ of ideals of various new rings $R^{\prime}$ constructed from $R$, in particular, the ring $S^{-1} R$ of fractions and the ring $R[X]$ of polynomials. For any partially ordered set $P$, we construct another poset $\mathbf{N}(P)$ and show that $P$ satisfies the ascending chain condition if and only if $\mathbf{N}(P)$ satisfies the ascending chain condition. As an application of this result, we give an order version proof for Hilbert's Basis Theorem.
\end{abstract}

\section{Introduction}

From a given commutative ring $R$, one can construct new rings in a number of standard ways, such as quotient rings, rings of fractions and the ring $R[X]$ of polynomials. The set $\operatorname{Idl}(R)$ of all ideals of $R$ is a complete lattice with respect to the inclusion order. Since Emmy Noether and her school's series of work, the lattice $\operatorname{Idl}(R)$ has been regarded as the most important order structure associated to the ring $R$. For instance, the definitions of both Noetherian rings and Artinian rings can be characterized in terms of the order structures of $(\operatorname{Idl}(R), \subseteq)$; a ring $R$ is arithmetical if and only if $(\operatorname{Idl}(R), \subseteq)$ is a distributive lattice [5] [2]. When we have a new ring $R^{\prime}$ constructed from a given ring $R$, a naturally raised question would be: how are the lattices $\operatorname{Idl}(R)$ and $\operatorname{Idl}\left(R^{\prime}\right)$ linked?

It is well known that for any ideal $I$ of a commutative ring $R$, there is a one-to-one correspondence between the ideals of the quotient ring $R / I$ and the ideals $J$ of $R$ containing $I$, that is, the poset $\operatorname{Idl}(R / I)$ is isomorphic to the principal filter $\uparrow I$ of $\operatorname{Idl}(R)$. Also, for any multiplicatively closed subset $S$ of $R$ (that is, $1_{R} \in S$ and $s_{1}, s_{2} \in S$ imply $\left.s_{1} s_{2} \in S\right)$, the lattice $\operatorname{Idl}\left(S^{-1} R\right)$ of ideals of the ring of fractions determined by $S$ is isomorphic to a subposet of $\operatorname{Idl}(R)$ through the mapping $h^{-1}: \operatorname{Idl}\left(S^{-1} R\right) \rightarrow \operatorname{Idl}(R)$, where $h: R \rightarrow S^{-1} R$ is the natural

2010 Mathematics Subject Classification. 06A06, 13E05, 13E99.

Key words and phrases. ring of fractions, multiplicatively closed subset, full m-closed set, ascending chain condition, Hilbert's Basis Theorem.

Received June 12, 2014; revised October 28, 2014. 
homomorphism which sends $x \in R$ to $\frac{x}{1_{R}}$ (see Lemma 5.24 of [7]). Thus $\operatorname{Idl}\left(S^{-1} R\right)$ is isomorphic to a subposet of $\operatorname{Idl}(R)$ which is closed under arbitrary meets. It seems, however, that there is still no characterization of subsets of $\operatorname{Idl}(R)$ that correspond to the set of ideals of the ring of fractions $S^{-1} R$ for some multiplicatively closed subset $S$ of $R$.

On the other hand, there generally does not exist order-embedding of $\operatorname{Idl}(R[X])$ into $\operatorname{Idl}(R)$, simply because $\operatorname{Idl}(R[X])$ usually has much more complicated and richer contents than $\operatorname{Idl}(R)$ does. For example, when $K$ is a field, $\operatorname{Idl}(K)$ only has two elements, while $\operatorname{Idl}(K[X])$ contains infinitely many elements.

In this paper, we first characterize the subposets of $\operatorname{Idl}(R)$ which are isomorphic to $\operatorname{Idl}\left(S^{-1} R\right)$ for some multiplicative closed set $S$ of a commutative ring $R$. Next we construct a partially ordered set $\mathbf{N}(P)$ from any given poset $P$, and show that there is a weak form of order embedding $G: \operatorname{Idl}(R[X]) \rightarrow \mathbf{N}(\operatorname{Idl}(R))$; this $G$, when restricted to any sub-chain of $\operatorname{Idl}(R[X])$, is an order-embedding. It is then proved that a poset $P$ satisfies the ascending chain condition if and only if $\mathbf{N}(P)$ satisfies the ascending chain condition. As an application, we give an order version proof for Hilbert's Basis Theorem.

All the rings considered in this paper are commutative rings with identity. The identity of $R$ will be denoted by $1_{R}$.

\section{Ideals of rings of fractions}

Let $R$ be a commutative ring and let $S^{-1} R$ be the ring of fractions determined by a multiplicatively closed subset $S$ (also called an m-closed subset) of $R$. Let $h: R \rightarrow S^{-1} R$ be the natural ring homomorphism, where $h(x)=\frac{x}{1_{R}}$ for each $x \in R . \quad$ For any $\mathscr{I} \in \operatorname{Idl}\left(S^{-1} R\right), \quad h^{-1}(\mathscr{I}) \in \operatorname{Idl}(R)$. Let $\operatorname{Idl}_{S}(R)=\left\{h^{-1}(\mathscr{I}): \mathscr{I} \in \operatorname{Idl}\left(S^{-1} R\right)\right\} . \quad$ Then $\operatorname{Idl}_{S}(R)$ is a subposet of $\operatorname{Idl}(R)$. The mapping $h^{-1}: \operatorname{Idl}\left(S^{-1} R\right) \rightarrow \operatorname{Idl}(R)$ is an order embedding [7], thus $\operatorname{Idl}_{S}(R)$ is order isomorphic to $\operatorname{Idl}\left(S^{-1} R\right)$. One natural converse problem is: given a collection $\mathscr{A}$ of ideals of $R$, under what conditions is there an m-closed set $S$ of $R$, such that $\mathscr{A}=\operatorname{Idl}_{S}(R)$ ?

Proposition 2.1. Let $S$ be an $m$-closed subset of the commutative ring $R$. The following statements are equivalent for an ideal $I$ of $R$ :

(1) $I \in \operatorname{Idl}_{S}(R)$.

(2) For every $x \in R$ and $s \in S$,

$$
s x \in I \text { implies } x \in I \text {. }
$$

Proof. Let $h: R \rightarrow S^{-1} R$ be the natural ring homomorphism. For any $J \in \operatorname{Idl}(R)$, let $J^{e}=\langle h(J)\rangle$ be the ideal of $S^{-1} R$ generated by $h(J)$, called the extension of $J$ to $S^{-1} R$. 
If $I \in \operatorname{Idl}_{S}(R)$, then there is an ideal $\mathscr{I}$ of $S^{-1} R$ such that $I=h^{-1}(\mathscr{I})$, which implies that $I=h^{-1}\left(I^{e}\right)$. Now if $x \in R$ and $s \in S$ such that $s x \in I$, then $h(x)=\frac{x}{1_{R}}=\frac{a}{s} \in I^{e}$, where $a=s x$. Hence $x \in h^{-1}\left(I^{e}\right)=I$. So (1) implies (2).

Now let $I$ satisfy the condition in (2). We prove that $I=h^{-1}\left(I^{e}\right)$, showing that $I \in \operatorname{Idl}_{S}(R)$. Suppose $x \in h^{-1}\left(I^{e}\right)$, then $h(x) \in I^{e}$, i.e. $\frac{x}{1_{R}}=\frac{a}{S}$ for some $a \in I$ and $s \in S$. Thus there is an $u \in S$ such that $u s x=u a$. Put $s^{\prime}=u s$. Then $s^{\prime} \in S$ and $s^{\prime} x=u a \in I$. Hence $x \in I$ by the assumption. Thus $h^{-1}\left(I^{e}\right) \subseteq I$. As the converse inclusion is always true, thus $I=h^{-1}\left(I^{e}\right)$.

It follows that (1) and (2) are equivalent.

COROLlary 2.2. If $\left\{I_{k}\right\}_{k \in M} \subseteq \operatorname{Idl}_{S}(R)$ then

$$
\bigcap_{k \in M} I_{k} \in \operatorname{Idl}_{S}(R)
$$

COROLlaRY 2.3. If $\mathscr{B}$ is a directed family of members of $\operatorname{Idl}_{S}(R)$ (for any $J, K \in \mathscr{B}$ there is $H \in \mathscr{B}$ such that $J \subseteq H, K \subseteq H$ ), then

$$
\bigcup_{I \in \mathscr{B}} I \in \operatorname{Idl}_{S}(R) \text {. }
$$

Corollary 2.4. If $I \in \operatorname{Idl}_{S}(R)$ then $\sqrt{I} \in \operatorname{Idl}_{S}(R)$.

Proof. Suppose $s x \in \sqrt{I}$ with $s \in S$ and $x \in R$. Then there is an $n \in \mathbf{Z}^{+}$ such that $(s x)^{n} \in I$, so $s^{n} x^{n} \in I$. As $s^{n} \in S$ and $I \in \operatorname{Idl}_{S}(R)$, by Proposition 2.1, we have $x^{n} \in I$, i.e. $x \in \sqrt{I}$. All these show, again by Proposition 2.1, that $\sqrt{I} \in \operatorname{Idl}_{S}(R)$.

From the above discussion, it is seen that for any m-closed set $S$ of $R$, the family $\mathscr{A}=\operatorname{Idl}_{S}(R)$ satisfies the following conditions:

(1) $R \in \mathscr{A}$

(2) $\mathscr{A}$ is closed under arbitrary intersections;

(3) $\mathscr{A}$ is closed under unions of directed subfamilies;

(4) $\mathscr{A}$ is closed under taking radicals of ideals.

Now given a family $\mathscr{A}$ of ideals of $R$ satisfying the conditions (1) to (4) above, must there be an m-closed set $S$ of $R$ such that $\mathscr{A}=\operatorname{Idl}_{S}(R)$ ? In the next section, we will answer this question.

\section{More characterizations of families $\operatorname{Idl}_{S}(R)$}

In this section, we prove a different characterization of $\operatorname{Idl}_{S}(R)$ for m-closed sets $S$ of a commutative ring $R$. A more concrete characterization for the 
ring of integers (actually for every principal ideal domain) will be derived. A negative answer is given to the problem posed at the end of last section.

Let $E$ be a subset of a commutative ring $R$. Define

$$
\Psi(E)=\{I \in \operatorname{Idl}(R) \text { : for any } x \in R \text { and } t \in E, t x \in I \text { implies } x \in I\} .
$$

Also for any collection $\mathscr{A}$ of ideals of $R$, define

$$
\Phi(\mathscr{A})=\{s \in R \text { : for any } I \in \mathscr{A} \text { and } x \in R, s x \in I \text { implies } x \in I\} .
$$

Remark 3.1. (1) For every $\mathscr{A} \subseteq \operatorname{Idl}(R), S=\Phi(\mathscr{A})$ is closed under multiplication and contains the identity $1_{R}$.

(2) Let $s_{1}, s_{2} \in R$ be any two elements in $R$ such that $s_{1} s_{2} \in S=\Phi(\mathscr{A})$. For any $I \in \mathscr{A}$ and $x \in R$, if $s_{2} x \in I$, then $s_{1} s_{2} x \in I$ because $I$ is an ideal, which then implies $x \in I$, therefore $s_{2} \in S$. Similarly, $s_{1} \in S$. It follows that $S=\Phi(\mathscr{A})$ satisfies the following property:

For any $s, t \in R, s t \in S$ if and only if $s \in S$ and $t \in S$.

In particular, if $s^{n} \in S$ for some positive integer $n$, then $s \in S$.

(3) For any $E \subseteq R, R \in \Psi(E)$ and $\Psi(E)$ is closed under arbitrary intersections and unions of directed subfamilies.

(4) For every $E \subseteq R$ and $\mathscr{A} \subseteq \operatorname{Idl}(R)$,

$$
E \subseteq \Phi(\Psi(E)), \quad \mathscr{A} \subseteq \Psi(\Phi(\mathscr{A})) .
$$

If $E \subseteq D$, then $\Psi(D) \subseteq \Psi(E)$. Similarly, if $\mathscr{A} \subseteq \mathscr{B}$ then $\Phi(\mathscr{B}) \subseteq \Phi(\mathscr{A})$.

Thus $\Psi$ and $\Phi$ define a Galois connection between the power sets of $R$ and that of $\operatorname{Idl}(R)$. It follows from the general properties of a Galois connection, that for any subset $E$ of $R$ and any subset $\mathscr{A}$ of $\operatorname{Idl}(R), \Psi(\Phi(\Psi(E)))=\Psi(E)$ and $\Phi(\Psi(\Phi(\mathscr{A})))=\Phi(\mathscr{A})$.

Proposition 3.2. (1) Let $\mathscr{A} \subseteq \operatorname{Idl}(R)$. Then $\mathscr{A}=\operatorname{Idl}_{S}(R)$ for some $m$ closed set $S$ if and only if $\Psi(\Phi(\mathscr{A}))=\mathscr{A}$.

(2) Let $S$ be an $m$-closed set of $R$. Then there is an $\mathscr{A} \subseteq \operatorname{Idl}(R)$ such that $S=\Phi(\mathscr{A})$ if and only if $S=\Phi(\Psi(S))$.

Proof. As the proofs of the two parts are similar, we just prove (1).

If $\Psi(\Phi(\mathscr{A}))=\mathscr{A}$, then $S=\Phi(\mathscr{A})$ is an m-closed set of $R$ and $\mathscr{A}=\operatorname{Idl}_{S}(R)$ by Proposition 2.1 .

Now assume that $\mathscr{A}=\operatorname{Idl}_{S}(R)$ for some m-closed set $S$ of $R$. Then $\mathscr{A}=\Psi(S)$, by Proposition 2.1. By Remark 3.1(4), $\Psi(\Phi(\mathscr{A}))=\Psi(\Phi(\Psi(S)))=$ $\Psi(S)=\mathscr{A}$.

An m-closed set $S$ of $R$ is called a full m-closed set if for any $x, y \in R, x y \in S$ if and only if $x \in S$ and $y \in S$. By Remark 3.1(2), $S=\Phi(\mathscr{A})$ is a full m-closed set of $R$ for any $\mathscr{A} \subseteq \operatorname{Idl}(R)$. 
For any m-closed set $S$ of $R$, there is a smallest full m-closed set containing $S$, which is the intersection of all full m-closed sets containing $S$.

Let $\hat{S}=\{x \in R$ : there exists $y \in R$ such that $x y \in S\}$. Then one can easily show that $\hat{S}$ is the smallest full m-closed set containing $S$.

The following proposition shows that every ring of fractions is defined by a full $\mathrm{m}$-closed set. $\hat{S}^{-1} R$.

Proposition 3.3. For any m-closed set $S$ of $R, S^{-1} R$ is isomorphic to

Proof. Define $f: S^{-1} R \rightarrow \hat{S}^{-1} R$ by $f\left(\frac{x}{s}\right)=\frac{x}{s}$. Clearly $f$ is a ring homomorphism. If $f\left(\frac{x}{s}\right)=f\left(\frac{y}{t}\right)$, then there exists $r \in \hat{S}$ such that $r t x=r s y$ holds in $R$. Now there exists $u \in R$ such that $u r \in S$, so urtx=ursy, implying $\frac{x}{S}=\frac{y}{t}$ as $u r \in S$. Thus $f$ is injective. For any $\frac{z}{v} \in \hat{S}^{-1} R$, there exists $u \in R$ such that $u v \in S$. Then $u \in \hat{S}$ and $\frac{z}{v}=\frac{u z}{u v}=f\left(\frac{u z}{u v}\right)$, showing that $f$ is surjective. Therefore $f$ is a ring isomorphism.

Using Proposition 2.1, we can derive a more concrete characterization of $\operatorname{Idl}_{S}(\mathbf{Z})$, where $\mathbf{Z}$ is the ring of integers.

Proposition 3.4. Let $\mathscr{A} \subseteq \operatorname{Idl}(\mathbf{Z})$ be a non-empty set with $\mathbf{Z} \in \mathscr{A}$. Then the following statements are equivalent:

(1) $\mathscr{A}=\operatorname{Idl}_{S}(\mathbf{Z})$ for some $m$-closed set $S$ of $\mathbf{Z}$.

(2) For any $I, J \in \operatorname{Idl}(\mathbf{Z}), I J \in \mathscr{A}$ if and only if $I \in \mathscr{A}$ and $J \in \mathscr{A}$.

Proof. (1) implies (2): Since (1) holds, $\mathscr{A}=\Psi(S)$ by Proposition 2.1. Note that for any $\langle a\rangle \in \operatorname{Idl}(\mathbf{Z}),\langle a\rangle \in \Psi(S)$ if and only if $\operatorname{gcd}(a, s)=1$ for all $s \in S$.

For any $I=\langle a\rangle$ and $J=\langle b\rangle, I J \in \mathscr{A}=\Psi(S)$ if and only if $\operatorname{gcd}(a b, s)=1$ for all $s \in S$, if and only if $\operatorname{gcd}(a, s)=1$ and $\operatorname{gcd}(b, s)=1$ for all $s \in S$. This is equivalent to $I=\langle a\rangle \in \mathscr{A}$ and $J=\langle b\rangle \in \mathscr{A}$.

(2) implies (1): By Proposition 3.2, it is enough to show $\Psi(\Phi(\mathscr{A}))=\mathscr{A}$. If $\mathscr{A}=\{\mathbf{Z}\}$, then $\Psi(\Phi(\mathscr{A}))=\mathscr{A}$ is clearly true. If $\{0\} \in \mathscr{A}$, then by (2), we can deduce that $\mathscr{A}=\operatorname{Idl}(\mathbf{Z})$ and so $\Psi(\Phi(\mathscr{A}))=\mathscr{A}$ also holds. Now we consider the case where $\mathscr{A} \neq\{\mathbf{Z}\}$ and $\{0\} \notin \mathscr{A}$. To show $\Psi(\Phi(\mathscr{A}))=\mathscr{A}$, we only need to check $\Psi(\Phi(\mathscr{A})) \subseteq \mathscr{A}$ since $\mathscr{A} \subseteq \Psi(\Phi(\mathscr{A}))$ always holds. Since (2) holds, $\langle a\rangle \in \mathscr{A}$ if and only if $\langle a\rangle=\mathbf{Z}$ or $\langle p\rangle \in \mathscr{A}$ for any prime factor $p$ of $a$. It follows that there is a set $E$ of positive prime integers such that

$$
\mathscr{A}=\left\{\left\langle p_{1}^{t_{1}} p_{2}^{t_{2}} \cdots p_{n}^{t_{n}}\right\rangle: p_{i} \in E, t_{i} \in \mathbf{Z} \text { and } t_{i} \geq 0, n \in \mathbf{Z}^{+}\right\} .
$$

(i) We claim that $a \in \Phi(\mathscr{A})$ iff $\langle a\rangle=\mathbf{Z}$ or $a$ has no prime factor in $E$. As a matter of fact, for any $a \in \Phi(\mathscr{A})$, suppose $\langle a\rangle \neq \mathbf{Z}$ and $a$ has a prime factor 
$p$ in $E$. So $a=a_{1} p$ for some $a_{1} \in \mathbf{Z}$. Then $\langle p\rangle \in \mathscr{A}$, and for any $s \in \mathbf{Z}$, $s a=s a_{1} p \in\langle p\rangle$ which implies $s \in\langle p\rangle$ by the definition of $\Phi(\mathscr{A})$. Therefore, $\mathbf{Z} \subseteq\langle p\rangle$, which is not possible. Conversely, if $\langle a\rangle=\mathbf{Z}$ or $a$ has no prime factor in $E$, then clearly $a \in \Phi(\mathscr{A})$.

(ii) Let $\langle m\rangle \in \Psi(\Phi(\mathscr{A}))$ and $\langle m\rangle \neq \mathbf{Z}$. We show that all the prime factors of $m$ are in $E$, therefore $\langle m\rangle \in \mathscr{A}$. Suppose $m=p m_{1}$, with $p$ a positive prime integer such that $p \notin E$. Then $p \in \Phi(\mathscr{A})$ by (i). Now $p m_{1} \in\langle m\rangle$. So $m_{1} \in$ $\langle m\rangle$ since $\langle m\rangle \in \Psi(\Phi(\mathscr{A}))$. Hence there is a $t \in \mathbf{Z}$ such that $m_{1}=t m=t p m_{1}$ which implies $p=1$, a contradiction. All these show that $\langle m\rangle \in \mathscr{A}$. Hence $\Psi(\Phi(\mathscr{A})) \subseteq \mathscr{A}$.

Remark 3.5. From the above proof, we can see that the above proposition also holds for every principal ideal domain (where the prime numbers are replaced by prime elements).

Example 3.6. Consider $\mathscr{A}=\{\langle 0\rangle,\langle 4\rangle,\langle 2\rangle,\langle 1\rangle\}$. Then $\mathscr{A}$ is a family of ideals of $\mathbf{Z}$ and satisfies the four properties listed at the end of Section 2. Now $\langle 4\rangle \in \mathscr{A}$ but $\langle 4\rangle^{2}=\langle 16\rangle \notin \mathscr{A}$. By Proposition $3.4, \mathscr{A} \neq \operatorname{Idl}_{S}(\mathbf{Z})$ for any $\mathrm{m}$ closed set $S$.

Proposition 3.7. An m-closed subset $S$ of $\mathbf{Z}$ is full if and only if $S=$ $\Phi(\Psi(S))$.

Proof. If $S=\Phi(\Psi(S))$, then by Remark 3.1 (1) and (2), $S$ is full.

Now let $S$ be full. Then $a \in S$ implies that each prime factor of $a$ is in $S$. It follows that there is a set $E$ of positive prime integers such that

$$
S=\left\{ \pm p_{1}^{t_{1}} p_{2}^{t_{2}} \cdots p_{n}^{t_{n}}: p_{i} \in E, t_{i} \in \mathbf{Z} \text { and } t_{i} \geq 0, n \in \mathbf{Z}^{+}\right\}
$$

One can then deduce that $\langle m\rangle \in \Psi(S)$ iff $\langle m\rangle=\mathbf{Z}$ or $m$ has no prime factor in $E$.

Let $x \in \Phi(\Psi(S))$. If $x$ has a positive prime factor, say $p$, such that $p \notin E$, then $\langle p\rangle \in \Psi(S)$. For any $s \in E, x s \in\langle p\rangle$, thus $s \in\langle p\rangle$ since $x \in \Phi(\Psi(S))$, which implies $s=p$, a contradiction. All these show that $x \in S$. So $\Phi(\Psi(S)) \subseteq S$, implying $S=\Phi(\Psi(S))$ since $S \subseteq \Phi(\Psi(S))$ always holds.

We still do not know the answer of the following problem.

Problem. Is it true that for any full m-closed set $S$ of a commutative ring $R, S=\Phi(\Psi(S))$ ?

\section{Posets satisfying the ascending chain condition}

In the following, by a poset (an abbreviation for partially ordered set) we mean a non-empty set $P$ equipped with a partial order " $\leq$ " that is reflexive, transitive and antisymmetric. 
For a subset $B$ of $P$, the supremum (infimum) of $B$ in $P$, denoted by sup $B$ (inf $B)$ or $\bigvee B(\bigwedge B)$ if it exists, is the least upper bound (greatest lower bound) of $B$. For more about posets and lattices, see [1] [3] [4].

An ascending chain in $P$ is a sequence $\left\{a_{i}: i \in \mathbf{Z}^{+}\right\}$of elements such that

$$
a_{1} \leq a_{2} \leq \cdots \leq a_{i} \leq \cdots .
$$

A descending chain in $P$ is a sequence of elements $\left\{a_{i}: i \in \mathbf{Z}^{+}\right\}$such that

$$
a_{1} \geq a_{2} \geq \cdots \geq a_{i} \geq \cdots .
$$

A poset $P$ is said to satisfy the ascending chain condition (or ACC for short) if for any ascending chain $\left\{a_{i}: i \in \mathbf{Z}^{+}\right\}$in $P$, there is $i_{0}$ such that $a_{k}=a_{i_{0}}$ for all $k \geq i_{0}$. The dual notion is the descending chain condition (DCC).

Example 4.1. (1) If $R$ is a commutative ring and $\operatorname{Idl}(R)$ is the poset of all ideals ordered by inclusion, then $R$ is Noetherian if and only if $\operatorname{Idl}(R)$ satisfies ACC. Dually, $R$ is Artinian if and only if $\operatorname{Idl}(R)$ satisfies DCC.

(2) Let $V$ be a vector space and $(\operatorname{Sub}(V), \subseteq)$ be the poset of all subspaces of $V$ ordered by inclusion. Then $V$ is finite dimensional if and only if $\operatorname{Sub}(V)$ satisfies ACC.

(3) Let $G$ be a group and $(\operatorname{Sub}(G), \subseteq)$ be the poset of all subgroups of $G$ ordered by inclusion. Then every subgroup of $G$ is finitely generated if and only if $\operatorname{Sub}(G)$ satisfies ACC.

(4) Let $M$ be an $R$-module with $R$ a commutative $\operatorname{ring}$ and $(\operatorname{Sub}(M), \subseteq)$ be the poset of all submodules of $M$ ordered by inclusion. Then $M$ is Noetherian if and only if $\operatorname{Sub}(M)$ satisfies ACC.

An ideal $I$ of a poset $P$ is a nonempty subset of $P$ satisfying the following:

(i) For any $x \in P$ and $y \in I$, if $x \leq y$, then $x \in I$. $y \leq z$.

(ii) For any two elements $x, y$ in $I$, there is a $z \in I$ such that $x \leq z$ and

Let $\operatorname{Idl}(P)$ denote the poset of all ideals of $P$ ordered by inclusion. The following results can be easily verified.

Proposition 4.2. (1) If $P$ satisfies $A C C$ (DCC resp.) and $P_{1}$ is a subposet of $P$, then $P_{1}$ satisfies ACC (DCC resp.).

(2) If $P$ and $Q$ satisfy $A C C$ (DCC resp.) then the cartesian product $P \times Q$ also satisfies $A C C$ (DCC resp.).

(3) A poset $P$ satisfies $A C C$ if and only if $(\operatorname{Idl}(P), \subseteq)$ satisfies $A C C$.

A monotone map $f: P \rightarrow Q$ between posets is a mapping such that $f(a) \leq$ $f(b)$ holds in $Q$ for any $a \leq b$ in $P$. A mapping $f: P \rightarrow Q$ is an order embedding if for any $a, b \in P, f(a) \leq f(b)$ if and only if $a \leq b$. 
Example 4.3. (1) Let $f: R \rightarrow A$ be a surjective ring homomorphism. Then

$$
f^{-1}: \operatorname{Idl}(A) \rightarrow \operatorname{Idl}(R)
$$

is an order embedding, where $f^{-1}$ sends $I \in \operatorname{Idl}(A)$ to $f^{-1}(I)$.

(2) Let $S$ be a multiplicatively closed subset of a commutative ring $R$ and $S^{-1} R$ be the ring of fractions of $R$. Let $h: R \rightarrow S^{-1} R$ be the natural ring homomorphism that sends $r \in R$ to $\frac{r}{1_{R}}$. Then $h^{-1}: \operatorname{Idl}\left(S^{-1} R\right) \rightarrow \operatorname{Idl}(R)$ is an order embedding (see [7], Lemma 5.24).

Definition 4.4. A monotone map $f: P \rightarrow Q$ between two posets is called a pre-order embedding if for any $a, b \in P, a \leq b$ and $f(a)=f(b)$ imply $a=b$.

Example 4.5. (1) Every order embedding is a pre-order embedding.

(2) Let $\mathscr{I}(\mathbf{R})$ be the set of all open intervals of $\mathbf{R}$ ordered by inclusion. Define $f: \mathscr{I}(\mathbf{R}) \rightarrow \mathbf{R}$ by $f((a, b))=b-a$. Then $f$ is a pre-order embedding that is not injective.

(3) Let $L=\{(x, y): x, y \in \mathbf{R}, x \geq 0, y \geq 0\}$ with the pointwise order. Define $g: L \rightarrow \mathbf{R}$ by $g(a, b)=\sqrt{a^{2}+b^{2}}$. Then $g$ is a pre-order embedding.

(4) Let $\left(\operatorname{Sub}_{\text {fin }}(V), \subseteq\right)$ be the poset of all the subspaces of finite dimensions of a vector space $V$. Define $g: \operatorname{Sub}_{\text {fin }}(V) \rightarrow E$ by $g(W)=\operatorname{dim}(W)$ for each $W \in \operatorname{Sub}_{\text {fin }}(V)$, where $E=\{0,1,2, \ldots\}$ is equipped with the ordinary order of numbers. Then $g$ is a pre-order embedding.

The following lemma follows directly from the respective definitions.

Lemma 4.6. If $g: P \rightarrow Q$ is a pre-order embedding and $Q$ satisfies $A C C$ (DCC resp.), then $P$ also satisfies ACC (DCC resp.).

\section{The monotone extension $\mathbf{N}(P)$ of $P$}

Let $\mathbf{N}$ be the set of non-negative integers with the ordinary order of numbers.

In this section, we construct a poset $\mathbf{N}(P)$ from any given poset $P$, called the monotone extension of $P$, and show that $P$ satisfies ACC if and only if $\mathbf{N}(P)$ satisfies ACC.

Definition 5.1. For any poset $P$, let $\mathbf{N}(P)$ be the poset of all monotone mappings $f: \mathbf{N} \rightarrow P$ with the order $\leq$ defined by $f \leq g$ iff $f(n) \leq g(n)$ for every $n \in \mathbf{N}$.

In the following, we shall use $\mathbf{N}(P)$ to denote the poset $(\mathbf{N}(P), \leq)$.

Remark 5.2. It can be verified that if $P$ is a meet (join) semilattice, then $\mathbf{N}(P)$ is also a meet (join) semilattice, where $f \wedge g$ and $f \vee g$ are defined by 
$(f \wedge g)(n)=f(n) \wedge g(n)$ and $(f \vee g)(n)=f(n) \vee g(n)$ for every $n \in \mathbf{N}$ respectively. Furthermore, if $P$ is a distributive (modular) lattice, then $\mathbf{N}(P)$ is also a distributive (modular) lattice.

For any poset $P$, define $F_{P}: P \rightarrow \mathbf{N}(P)$ by

$$
F_{P}(a): \mathbf{N} \rightarrow P, \text { for all } a \in P,
$$

where $\left(F_{P}(a)\right)(n) \equiv a$ is the constant mapping with value $a$.

Define $G_{P}: \mathbf{N}(P) \rightarrow P$ by $G_{P}(f)=f(0)$ for each $f \in \mathbf{N}(P)$.

Then both $F_{P}$ and $G_{P}$ are monotone, and for any $a \in P$ and $f \in \mathbf{N}(P)$,

$$
G_{P}\left(F_{P}(a)\right)=a \quad \text { and } \quad F_{P}\left(G_{P}(f)\right) \leq f .
$$

Obviously $G_{P}$ is surjective and $F_{P}$ is an order embedding.

Theorem 5.3. For any poset $P, P$ satisfies $A C C$ if and only if $\mathbf{N}(P)$ satisfies $A C C$. 4.6 .

Proof. Since $F_{P}$ is an order-embedding, the sufficiency follows from Lemma

Now suppose that $P$ satisfies ACC. Let

$$
f_{1} \leq f_{2} \leq \cdots \leq f_{n} \leq \cdots
$$

be an ascending chain in $\mathbf{N}(P)$. Note that if $n \leq m$ then $f_{n}(n) \leq f_{n}(m) \leq f_{m}(m)$, so the following is an ascending chain in $P$ :

$$
f_{1}(1) \leq f_{2}(2) \leq \cdots \leq f_{n}(n) \leq \cdots .
$$

By the assumption on $P$, there is an $m$ such that $f_{k}(k)=f_{m}(m)$ whenever $k \geq m$. Now for each $i \leq m$,

$$
f_{1}(i) \leq f_{2}(i) \leq \cdots \leq f_{k}(i) \leq \cdots
$$

is an ascending chain in $P$, so there is a $t_{i}$ such that $f_{k}(i)=f_{t_{i}}(i)$ for all $k \geq t_{i}$. Let $t=\max \left\{t_{1}, t_{2}, \ldots, t_{m}, m\right\}$.

We claim that $f_{k}=f_{t}$ for all $k \geq t$.

As a matter of fact, for any $l \in \mathbf{N}$,

(i) if $l \leq m$ then, as $t_{l} \leq t \leq k, f_{k}(l)=f_{t_{l}}(l)=f_{t}(l)$;

(ii) if $m<l$, then $f_{m}(m) \leq f_{m}(l) \leq f_{t}(l) \leq f_{k}(l) \leq f_{s}(s)=f_{m}(m)$, where $s=$ $\max \{l, k\}$. The last equation holds because $m \leq s$. This again shows that $f_{k}(l)=f_{m}(m)=f_{t}(l)$.

All these show that $f_{k}=f_{t}$.

Thus $\mathbf{N}(P)$ satisfies ACC.

Lemma 5.4. Let $R$ be a commutative ring. Then there is a pre-order embedding

$$
G: \operatorname{Idl}(R[X]) \rightarrow \mathbf{N}(\operatorname{Idl}(R))
$$


Proof. Define the mapping $G: \operatorname{Idl}(R[X]) \rightarrow \mathbf{N}(\operatorname{Idl}(R))$ as follows: for any ideal $\mathscr{I}$ of $R[X]$ and $i \in \mathbf{N}$,

$$
G(\mathscr{I})(i)=\left\{a \in R: \text { there exists } a_{0}+a_{1} X+\cdots+a_{i-1} X^{i-1}+a X^{i} \in \mathscr{I}\right\} .
$$

Then Lemma 8.6 of [7] says exactly that $G$ is a pre-order embedding.

Corollary 5.5 (Hilbert's Basis Theorem). Let $R$ be a commutative Noetherian ring and $X$ be an indeterminant. Then the ring $R[X]$ of polynomials is again a Noetherian ring.

\title{
REFERENCES
}

[ 1 ] B. A. Davey and H. A. Priestley, Introduction to lattices and order, Cambridge University Press, Cambridge, United Kingdom, 2002.

[2] L. Fuchs, Über die Ideale arithmetischer Ringe, Comment. Math. Helv. 23 (1949), 334-341.

[3] G. Gierz et AL., Continuous lattices and domains, Encyclopedia of mathematics and its applications 93, Cambridge University Press, Cambridge, United Kingdom, 2003.

[4] G. Grätzer, General lattice theory, Birkhäuser Verlag, CH-4010 Basel, Switzerland, 2003.

[5] C. U. Jensen, Arithmetical rings, Acta. Math. Acad. Sci. Hung. 17 (1966), 115-123.

[6] G. C. Rota, The many lives of lattice theory, preprint.

[7] R. Y. Sharp, Steps in commutative algebra, London Math. Society student texts 19, Cambridge University Press, Cambridge, United Kingdom, 1990.

\author{
Yuan Ting Nai \\ Mathematics and Mathematics Education \\ National Institute of Education \\ Nanyang Technological University \\ 1 Nanyang Walk, 637616 \\ SINGAPORE \\ E-mail: 9naiyt@gmail.com \\ Dongsheng Zhao \\ Mathematics and Mathematics Education \\ NATIONAL INSTITUTE OF EDUCATION \\ Nanyang Technological University \\ 1 Nanyang Walk, 637616 \\ SINGAPORE \\ E-mail: dongsheng.zhao@nie.edu.sg
}

